\title{
New Trends in Learning about Disaster Prevention: Some Lessons from the 2011 Great East Japan Earthquake
}

\author{
Naoko Suzuki \\ University of Tokushima, Japan
}

\begin{abstract}
Lying on one of the world's most seismologically active areas, Japan has been affected by frequent natural disasters. In fact, many lessons have been learned after each occurrence of a horrible event from the ancient times up to the present, which have been implemented in order to avoid and/or reduce the degree to which the country is affected by such disasters. Yet, until the recent years, these lessons tended to be diffused within local communities only and thus remained within a narrow and closed society. However, the Great East Japan Earthquake in March 2011, one of the most serious catastrophes of the last 1000 years, has gradually been changing this tendency and has prompted a wide variety of new learning styles. One of the most conspicuous features of this is the enhancement of mutual learning at various levels and through different methods between those who were the most seriously affected and those who, no matter how near or far from the affected area they live, are worried about future disasters of this kind which have been predicted. Thus the recent disaster has had positive impacts on learning about disaster prevention among the nationwide population, generating a variety of new approaches. This paper intends to illustrate some good examples of new trends in learning about disaster prevention and indicate how the nation has been transferring the experience of catastrophes into effective learning.
\end{abstract}

\section{Introduction}

Most nations in the world which geologically lie on the Circum-Pacific earthquake zone, one of the world's most seismologically active areas, have been more or less experiencing earthquakes, tsunamis, typhoons, flooding, landslides, volcanic eruptions and other natural disasters. Among these nations, seismological discussions are often held, covering the issue from a wide range of angles. Since Japan is one of these countries where frequent natural disasters are unavoidable, it always has to be well prepared for all kinds of disasters as well as improve its mitigation skills, whilst investing a large amount of funds into disaster-related research. Moreover, the geographical features of the country, which comprises four main islands and a number of smaller islands with an undulating landscape and a deeply- indented coastline, sometimes enlarge the scale of each disaster.

Although the nation has been earnestly taking all possible measures to reduce the influence of all assumable natural disasters, the earthquake which happened on 11th of March, 2011 (the 2011 Great East Japan Earthquake) and the following tsunami were greater than previously expected, and 15,885 people died and 2623 remain missing as of 10th of April 2014 [1]. Furthermore, since the 2011 Great East Japan Earthquake triggered the collapse of nuclear power plant building No.1 in Fukushima, those who used to live in the areas that have now become the most contaminated have been forced to evacuate and there are still no prospects for them to go back home. Meanwhile, those who decided to continue living in surrounding areas which are close to the most contaminated areas will face the dangers of radiation for a long period of time. Also, due to the prolonged influence of radiation, the average period of living at the evacuation centres was longer than was the case in other disasters, even though the government has been putting an enormous effort into decontamination. All the evacuation centres had ceased their operations by 2014 , and all the people there have moved to provisional houses built after the earthquake, or other rented accommodation etc. According to the Reconstruction Agency, among those who have been directly affected by the 2011 disasters, 263,958 people have still been unable to return to their hometown and had scattered to live in other parts of the country as of 13th of March, 2014 [2]. Moreover, the number of disaster-related deaths had reached more than 2916 by 30 September 2013 [3]. Such deaths included those who committed suicide or died due to accumulated stress caused by prolonged living at the evacuation centres, loss of jobs and/or family members and harmful rumours about those areas.

In the immediate aftermath of the last disaster, the demand for study of the impacts of radiation on the human body and for future disaster preparedness rose sharply; however, concerns among people have gradually diverged in various directions over the last three years, moving to issues such as reflecting on the pros and cons of previous preparedness for the disasters. Discussions are taking place in diverse fields at various levels, involving some brand-new ideas to carve out the direction of a bright future. In terms of education for disaster preparedness and mitigation, the main discussions used to be held 
among researchers in the field of engineering [4], yet some discussions have begun to be held among educational researchers in recent years, as this field has begun to be treated as a cross-cutting research area. Although there is still a long way to go to recover entirely from the enormous damage to the whole country, the trends in learning spreading around the nation generated after the 2011 Great East Japan Earthquake at least promise a silver lining for the future. This paper intends to illustrate some good examples of new trends in learning about disaster prevention and indicate how the nation has been transferring the experience of catastrophes into effective learning.

\section{Some Historical Records of Learning about Disaster Prevention and the Current Utilization of Lessons from the Past}

Since the nation has experienced massive catastrophes throughout its history, learning at school is naturally subject to the influences of various disasters, apart from the ordinary subject curriculum. The record of lessons learned from previous disasters can be traced back to the old days, and some old sayings have remained in many parts of the nation, and those records can be seen on historical monuments such as gravestones. Nowadays, there are some conservation movements to retain these lessons from past disasters by making use of them in the classroom teaching at schools.

For instance, the most affected area of the 2011 Japan Earthquake, the coastline of Eastern Japan, is one of the places in the country where large scale earthquakes have occurred many times in history. The record of earthquakes in those areas can be traced back to the early 1600 s, and earthquakes whose scale exceeds magnitude 7.0 took place in 1611, 1677, 1763, 1793, 1856, 1896, 1897, 1933, 1968, 1994 and 2011 [5]. Among them, the earthquakes that happened in 1611, 1896, 1933 and 2011 coincided with massive tsunamis, resulting in a high number of dead and missing. In these areas, there is a tsunami folklore that has been handed down through generations called 'Tsunamitendenko' [6]. 'Tendenko' literally means 'spreading apart', and 'Tsunami-tendenko' can be interpreted as 'When you feel the earthquake on the coastline, you should evacuate from the possible site of tsunami to the upper areas of the land as soon as you can without worrying about others around you, no matter how close your relationship with them.' This also means that 'You should save yourself first and should not sacrifice yourself for others. This attitude, even though it appears to be showing the cold shoulder at first glance, will save millions of lives in the end'. Although this old legend has been established in those areas for a long period of time, whether or not one believes in this determined one's survival or death at the time of the 2011 disasters.

Following the idea of 'Tsunami-tendenko', in primary and junior high schools in Kamaishi City with a population of 37,909 as of December 2011 [7], located on the coastline of Iwate Prefecture, all the pupils had been taking a special training programme for eight years to prepare them for the dangers of earthquakes and tsunamis, under the supervision of Toshitaka Katada, Professor of Gunma University [8]. In this programme, children were trained not only to gain knowledge about disaster prevention, but also to learn how to protect their own lives. There are three main rules to follow: 'Don't be preoccupied by previous assumptions.' (For instance, children are taught that they should not believe in the current hazard map as it is, since natural disasters are unpredictable and often exceed previous assumptions); 'Do your best/hardest!' (This means that you should do as much as possible until you reach your limit; thereafter all you could do is to accept the outcome); and 'Be the first to evacuate.' (Originating from the idea of 'Tsunami-tendenko', this attitude eventually turns out to mean taking initiative in evacuation, since there is a psychological tendency for human beings to behave as if they are in a normal situation even when they should evacuate, unless someone takes a lead on this matter). In Kamaishi City, when the massive earthquake happened on the afternoon of 11th of March, 2011, all the children who were at school or even who were on the way back home after school immediately followed these rules without hearing any advice from others, and as a result, it was said that $99.8 \%$ of the children survived. In Kamaishi City as a whole, more than 1000 people died or went missing; however nearly 3000 children were able to escape from the tsunami and their attitudes even prompted adults around them to evacuate. This is called 'The miracle of Kamaishi', because in other affected areas where insufficient training had been given, many children became the victims of the tsunami. In fact, only $62 \%$ of schools in the most affected areas of three prefectures (Miyagi, Iwate and Fukushima) had conducted training programmes to reducing the effects of tsunamis before, while $94 \%$ of schools had introduced training programmes against earthquakes, according to the statistics of March 2012 [9].

In other parts of the whole country, similar old legends remained and in some cases were utilised or were to be utilised in education on disaster prevention. For instance, in Shikoku Island, a smaller island located in the south-western part of Japan, which has also experienced a number of natural disasters including floods, landslides, earthquakes, tsunamis, storm surges and drought in the past, there are more than 500 historical sayings about disasters, including tips and hints for survival, 
such as how to mitigate damages caused by the disaster, how to be prepared, how to cope after the event and how to recover from it, and today there are attempts to make use of these old legends in future educational programmes for disaster prevention so that people can improve their disaster mitigation skills [10].

In the past, these lessons, which were mostly passed down by word of mouth, tended to be diffused within local communities only and thus remained within a narrow and closed society. However, in recent years, particularly since the 2011 event, the lessons from the past events have been exchanged in wider areas no matter where one lives, whilst there is a tendency to retain these old sayings by collecting more precise information and analysing it for future reference.

\section{Some New Trends in Learning about Disaster Prevention}

The 2011 Great East Japan Earthquake has prompted the whole nation to reconsider many aspects of the industrial society, and learning is no exception. In terms of education, a large number of measures have been taken physically as well as mentally by various stakeholders at multi levels, and those can be observed not only in the affected area but also in other parts of the country. The following are some examples which have appeared since 2011.

Firstly, more effective use of social media, especially by way of the internet, has been developed to improve the efficiency of exchanging views among different kinds of likeminded people. Just after the 2011 earthquake and tsunami, everything was in confusion especially along the coastline of Eastern Japan, since most school buildings were heavily damaged and/or flooded, and therefore some children had to take classes at the evacuation centre or had to move to other schools [11]. 25,751 children moved to other schools in other parts of the whole country. 622 public schools were used as evacuation centres and in some schools teachers had to take the initiative in operating evacuation centres, as well as carrying out their ordinary teaching, since there were no instructions from the appropriate governmental office about how to proceed after the disaster. The Ministry of Education had set up the Headquarters of Educational Measures for the 2011 Disaster and from these headquarters, some members of staff were dispatched to the affected areas in order to assess the real circumstances and consider necessary action from an educational perspective. However, the greatest difficulty for them was the breakdown of all means of communication, including the use of mobile phones, making it hard to control and organise responses to the different necessities rising from many places. Due to this, it was impossible to establish communications between schools and local education boards and between local education boards and prefectural education boards. This caused a delay in full and immediate implementation of delivering aid and stock relief supplies, as normally local and prefectural education boards were supposed to take the primary roles in managing and coordinating these different needs.

In order to cope with these confusions and difficulties in communication, the Ministry of Education set up the website the 'Portal Site of the Great East Japan Earthquake and Learning for Children', where people can see who requires help and who wishes to help others [12]. On this website, a variety of aids and assistance were proposed from local and prefectural education boards, schools, companies, NPOs etc. from all over the nation, including not only physical assistance such as the provision of school supplies, equipment, and books, but also human assistance such as the dispatch of teachers and various educational experts such as counsellors and volunteers as well as the re-location of children who used to live in the affected areas. In more than 2000 cases there was successfully coordination between those who required help and those who wished to help others and approximately 300,000 visits in total were counted on that website. In addition, this website gave children an opportunity to express their own views and also provided them with information on special scholarship programmes offered by companies, public-interest corporations, and local governments. The website ceased its operations on 11th of May, 2012, as similar sites had appeared. Some of its roles such as providing information on scholarship programmes and matching support were taken over by the new website 'Recovery from the Great East Japan Earthquake' which was launched on 1st of May, 2012 [13]. Apart from the Ministry of Education, the National Institute for Educational Policy Research also began to operate the 'Site for supporting the operation of schools in the affected area' which mainly provides ideas and methodology of operating schools, instructions on teaching during an emergency and educational consultancy etc [14]. This site also collects and provides information such as how to teach effective energy saving and disaster prevention so it can be used not only in the affected areas but also in other parts of the country as a whole. Apart from these, a large number of unique websites of similar kinds have been introduced by private companies, international organisations, nonprofit organisations, volunteers and so forth.

In order to help with and promote these activities, some of the latest satellites introduced by the Japan Aerospace Exploration Agency (JAXA) have been widely utilised. For example, earth observing satellites, such as the Advanced Land Observing Satellite (ALOS), assisted the recovery process by providing the latest maps of the affected area for 
relevant ministries and local governments, utilizing advanced land-observing technology [15]. Also, considering the fact that all the means of communication were destroyed after the disaster, which had hindered the process of recovery, largevolume and high-speed communications provided by the KIZUNA (WINDS) were utilised in order to enhance communications between people who are in different places, even where major ground infrastructure for the Internet was difficult to set up [16]. The Engineering Test Satellite-VIII KIKU No.8 also contributed to the recovery by enhancing the environment for mobile-phone-based communications and contributing to the development of technologies for a satellite-based multimedia broadcasting system [17].

Secondly, as the recovery progressed, groundbreaking idea from all over the country tended to be implemented in the most affected areas of the 2011 disaster, since the experts in various different fields gathered and exchanged their cutting edge ideas to renovate those areas. One conspicuous example can be found in the Minamisouma Solar-agri Park, Fukushima Prefecture [18]. Minamisouma is a city in the coastal area of Fukushima Prefecture with a population of 71,000 including 6500 children, which was heavily damaged by the 2011 earthquake, tsunami and the spreading rumours of radiation. Approximately 600 people in the city died because of the tsunami, and the population decreased to less than 10,000 after the disaster since most people evacuated to other places. Although some people in the city moved to live in other parts of the country, some have returned and now 45,000 people including 3,000 children live in the city.

Minamisouma Solar-agri Park was established in March 2013 by Eijyu Hangai, who previously worked as an executive for the Tokyo Electric Company. He utilized about 2.3ha of flat land which was one of the areas most affected by the tsunami. The purpose of the park is to help children grow up healthily in Fukushima and to reduce the influence of harmful rumours prevailing across and beyond the nation by making a meeting place for people coming from different parts of the country. It is hoped that the park will also help in re-establishing trust and will contribute to the recovery in the lives of ordinary people and of industry in Fukushima through providing learning opportunities by making use of a photovoltaic station and two plant factories. In September 2011, 'the Solar Energy Company for the Recovery of Fukushima' was established, creating $500 \mathrm{~kW}$ electricity, utilising personal funds and subsidies from the Ministry of Agriculture, Forestry and Fisheries. In April 2012, 'the Association for the Utilisation of Photovoltaic Stations and Agriculture for the Recovery of Fukushima' was also established. These two organisations started to operate in Minamisouma
Solar-agri Park in March 2013. The main targets of the current programmes are primary and junior high school students, but it is planned to include senior students from high school and universities by offering an 'open school' at weekends from May 2014. Also, during the summer vacation, it is intended to provide a meeting place for all the people throughout the country. The electricity generated in this park is being used for two plant factories built next to the photovoltaic station, but the surplus is being sold to a local electric company. The two plant factories are operated by a local agricultural corporation called 'Izumi-New World', and all the lettuces produced in the factories are purchased by the local supermarket chain called 'York Benimal'.

Currently, the main operation of the park comprises planning and coordinating children's learning through the photovoltaic station and the two plant factories; dispatching the latest news about Minamisouma through the website in order to reduce the influence of harmful rumours; planning and coordinating programmes for meetings among different people during the summer vacation; creating job opportunities; and strengthening human resource development. In the current programme for primary and junior high school children which started in April 2013, a variety of unique learning opportunities have been introduced: understanding energy through studying familiar things; finding energy in plants and animals; experiencing the flow of air through a windmill and a parachute; experiencing the energy of light using an insect magnifier; converting energy to other things; experiencing aspects of the jobs in the photovoltaic station to understand how to produce energy by solar photovoltaic electrification; moving solar panels installed on a roof, charging an electric car; controlling the temperature and humidity of the plant factories by computer; harvesting lettuce in the plant factory by computer; understanding the latest technology of agriculture and considering future possibilities; understanding the global economy; and reflecting on the whole process and deepening learning etc. Through these interesting experiences, it is expected that children will develop skills of critical thinking, presentation skills, and behavioural abilities. The fees are free for children and all the costs of these programmes are covered by an endowment from the Nippon Foundation, the Mitsubishi Corporation Disaster Relief Foundation and other sponsoring bodies.

Thirdly, mutual learning has been developed in many parts of the country between those who have been seriously affected by the latest disaster and those who have not been affected but expect to experience something similar in the near future, or those who have been previously affected by a similar disaster. The learning has been in regard to how to reduce the effects of a disaster, how to operate an 
evacuation centre and how to treat the victims of the disaster etc.

In reaction to the 2011 disasters, the role of the schools and the content of education programmes for disaster prevention have been reconsidered at meetings of advisers in the Ministry of Education. Concerning the role of schools, it is now proposed that schools should always be prepared to become evacuation centres by enhancing their earthquake safety and resistance, including the renovation of non-structural elements of the whole building. According to statistics [19], although about $90 \%$ of public schools established by local governments are designated as evacuation centres, the number of schools which normally build stockpiles of materials such as emergency kits (batteries, battery chargers, torches, radios, candle lanterns, thermal sheets, gas cylinders, portable gas stoves etc.), emergency foods and water, toiletries, sanitation supplies and equipment, and drugs is relatively low, which would make it difficult for most schools to operate evacuation centres appropriately. In addition, it is proposed that proper training should be required not only for children, but also for teachers, so that they can understand all the possible influences of the disasters from various aspects, and take appropriate measures immediately during an emergency. Concerning the contents of education programmes for disaster prevention, the Ministry of Education issued 'Guidelines for creating school manuals for disaster prevention' in March 2012 [20], including a new methodology of teaching disaster prevention which attaches importance to creative and subjective ability to think and act.

Following these proposals of the central government, Tokushima Prefecture in Shikoku Island has vigorously taken all safety precautions and implemented some effective measures. This is because along the coastline of south-western Japan including Tokushima, large-scale devastating earthquakes and tsunami hit in 1707, 1854 and 1946 [21], and it is predicted that a massive earthquake is highly likely to happen within the next 30 years. The education board of the prefectural government have revised the contents of existing school manuals on disaster prevention, following the national guidelines issued in 2012, and have examined the areas expected to be flooded in the next tsunami. At the same time, the prefectural education board has proposed the following: to revise the contents of education programmes at school on disaster prevention in order to save more lives; to set up a club for disaster prevention at each high school which could take a role of training human resources for future disaster prevention; to enhance earthquake-resistance strength and enhance earthquake safety of school buildings; to improve some schools ability to fulfil the role as evacuation centres since they are designated as the main rally points at the time of a disaster; to ensure appropriate opportunities of learning about disaster prevention throughout one's life; to train specialists who can coordinate disaster-related issues such as an appropriate operation of the evacuation centre among schools, families and communities; and to raise awareness of the need for effective communication between people of different backgrounds during an emergency in a local community.

Sponsored by the Ministry of Education, the prefectural education board in Tokushima also coordinated a summer camping project at four schools, all of which are located in different parts of the prefecture [22]. This project was open to everyone who lived in each of the school areas and involved a variety of activities: participating in disaster drills; lectures on natural disasters given by researchers of Tokushima University, firefighters and members of the Self-Defence Forces; experiencing the life in an evacuation centre such as making a private room with a cardboard box, having emergency food, organising a soup-run, and staying overnight with people one does not know; walking along the evacuation route to check and make hazard maps; and reflecting on what they have learned through this camping project. At the seminar to review the project, an expert on disaster prevention was invited by Fukushima Prefecture. He had taken on the daunting task of operating one of the main evacuation centres in Fukushima since the 2011 Great Japan East Earthquake, and he gave valuable advice to each school in Tokushima. Through these events, other problems were revealed, such as how to treat those who require special care both mentally and physically. The expert invited from Fukushima had been greatly assisted during the 2011 disasters by those who took a leading role in recovery operations after the Niigata Chuetsu Earthquake of 2004, and therefore he acknowledged that it is the responsibility of those who have been affected by disasters to pass on the lessons they have learned to places which have a high probability of a future disaster [23]. In this way, mutual learning has been naturally fostered among people living all over the nation in recent years, especially between those who have been seriously affected by the latest disaster and those who have not been affected but expect to experience it in the near future or those who have been previously affected by a similar disaster.

\section{Concluding Remarks}

Although Japan has experienced massive natural disasters many times throughout its history, the impact of the 2011 Great East Japan Earthquake on the whole society is still enormous, not only in its visible damage caused by the devastating earthquake, tsunami and the collapse of a nuclear power plant, but also in its invisible influences which have had a 
psychological effect on many people who live in this country. Yet, the latter does not necessarily have to be seen from a pessimistic viewpoint, since it has given the nation great leverage to overcome the harsh conditions in the face of a perceived unprecedented crisis and tapped the great latent strength of the people in the process of recovery to reconsider the meanings of everything generated by the industrial society since its beginning. In the field of education, a variety of new proposals have been generated and some cutting edge ideas have been introduced that break the new ground in creating a better future. In terms of disaster prevention, whilst continuing to learn from historical records, the nation has been challenged to explore the potentiality of future learning approaches in many respects, such as considering more effective use of social media, introducing cutting edge ideas into education by utilising a photovoltaic station and plant factories, and enhancing the variety of mutual learning between people of different backgrounds. Furthermore, the 2011 event has more or less converted individual attitudes of mind toward natural disasters, and many people have become more conscious of how to be prepared for future disasters, how to behave in the case of an emergency and how to develop relationships with people in the community etc. Although there is still a long way to go before there is complete recovery from the 2011 disasters, these new trends in learning, generated from a profound lesson, show how this country should take a role in the future for the whole world.

\section{References}

[1] Emergency Headquarters (2014), 'The Extent of Damages caused by the Great East Japan Earthquake and Measures taken by the Police', National Police Agency; http://www.npa.go.jp/archive/keibi/biki/higaijokyo.pdf (11 April 2014)

[2] Reconstruction Agency (2014), 'The Number of Evacuees as of 13th of March 2014'; http://www.reconstruction.go.jp/topics/main-cat2/sub-cat21/20140327_hinansha.pdf (11 April 2014)

[3] Reconstruction Agency (2013), 'The Number of Disaster-related Death'; http://www.reconstruction.go.jp/ topics/main-cat2/sub-cat2-1/20131224_kanrenshi.pdf (11 April 2014)

[4] See for instance: Shaw, R., Shiwaku, K., Kobayashi, H. \& Kobayashi, M. (2004), 'Linking experience, education, perception and earthquake preparedness', Disaster Prevention and Management, Vol. 13 Iss: 1, pp.39 - 49.

[5] Headquarters for Earthquake Research Promotion (2011), 'The Long-term Evaluation of Seismic Activities in Waters off from Sanriku to Bousou Coasts (The Second Edition)', The special governmental organization attached to the Prime Minister's office (now belongs to the Ministry of Education, Culture, Sports, Science and Technology); http://www.jishin.go.jp/main/chousa/11nov_sanriku/sanrik u_boso_3_hyoka.pdf (11 March 2014)

[6] Tsuji, Y. (2014), 'The survival rate was 99.8\%: (The Miracle of Kamaishi): The Correctness of Tsunamitendenko', Sankei News;

http://sankei.jp.msn.com/science/news/140310/scn140310 09350003-n1.htm (11 April 2014)

[7] Kamaishi City (2012), 'Statistics of Kamaishi City in the Year of 2011', http://www.city.kamaishi.iwate.jp/ index.cfm/10,22134,c,html/22134/20121102-181956.pdf, p.12 (11 April 2014)

[8] Education Board of Kamaishi City, Division of Disaster Prevention within Kamaishi City Council \& Disaster Social Engineering Laboratory of Gunma University (2010), 'The Teaching Manuals for the Prevention of Tsunami in Kamaishi City' http://dsel.ce.gunma-u.ac.jp/kamaishi_tool/index.html (11 April 2014)

[9] Ministry of Education, Culture, Sports, Science and Technology (2012), 'Survey Report on Schools' Reactions to the Great East Japan Earthquake'; http://www.mext.go.jp/a_menu/kenko/anzen/_icsFiles/afi eldfile/2012/07/12/1323511_2.pdf, pp.64-65 (11 March 2014)

[10] Matsuo, Y., Wada, K., Yamamoto, M. and Nakano, S. (2010), 'A study on making good use of disaster mitigation skills included in the historical sayings of Shikoku region to raise ability for regional disaster prevention', Journal of Japan Society for Natural Disaster Science, Vol.29, No.3, pp.393-411.

[11] Ministry of Education, Culture, Sports, Science and Technology (2011), 'The 2011 White Paper Section 1: The Recovery from the Great East Japan Earthquake: Creative Recovery through Human Development'; http://www.mext.go.jp/b_menu/hakusho/html/hpab201201 /detail/1324384.htm\#contentsStart (11 March 2014)

[12] Ibid.

[13] Ministry of Education, Culture, Sports, Science and Technology (2014), 'Recovery from the Great East Japan Earthquake'; http://fukkokyoiku.mext.go.jp/ (11 March 2014)

[14] National Institute for Educational Policy Research (2014), 'Site for Supporting the Operation of Schools in the Affected Area'; http://www.hisaichi-gakkoushien.nier. go.jp/(11 March 2014)

[15] Earth Observation Research Center (1997), 'About ALOS - Overview and Objectives - ', Japan Aerospace Exploration Agency;

http://www.eorc.jaxa.jp/ALOS/en/about/about_index.htm (11 March 2014)

[16] Japan Aerospace Exploration Agency (2003), 'Overview of the KIZUNA';

http://global.jaxa.jp/countdown/f14/overview/kizuna_e.ht ml (11 March 2014) 
[17] Japan Aerospace Exploration Agency (2006), 'About Engineering Test Satellite VIII "KIKU No.8" (ETS-VIII)'; http://global.jaxa.jp/projects/sat/ets8/index.html March 2014)

[18] Tohoku Electric Company (2014), 'Report on the Recovery from the 2011 Great East Japan Earthquake', http://www.tohoku-epco.co.jp/fukyu/report/contents/ f48_aguripark/index.html (11 April 2014)

Minamisouma-Agri Park (2014), 'Outline of the Minamisouma-Agri Park'; http://minamisomasolaragripark.com/about (11 April 2014)

[19] Educational Facilities Research Center (2013), 'Actual Condition Survey on the Capability of Schools for Disaster Prevention', National Institute for Educational Policy Research; http://www.nier.go.jp/shisetsu/ pdf/bousaikinou2013.pdf (11 March 2014), p.2 \& p.6

[20] Ministry of Education, Culture, Sports, Science and Technology, (2012), 'The Guideline for Creating School Manuals for Disaster Prevention'; http://www.mext.go.jp/ a_menu/kenko/anzen/1323513.htm (11 March 2014)

[21] Otani, H., Murakami, H. \& Kouzuki, Y., (2003), 'Approach to inspection of damage of features by the 1946 Showa Nankai earthquake in Tokushima city', Historical Earthquakes, Vol.19, pp.139-145

[22] The Education Board of Tokushima Prefecture, (2014), 'Lifelong Learning Project on Disaster Prevention through Camping Experience' in the Leaflet of the Project issued by the Education Board of Tokushima Prefecture.

[23] Amano, K., (2013), 'Creating a community: Towards a society where people can feel that I am happy to be born here.' (Presentation at the seminar to review the camping, held on 5th of December, 2013), Organising Committee for the 'Lifelong Learning Project on Disaster Prevention through Camping Experience', Education Board of Tokushima Prefecture. 